\title{
CHAOS AND STABILITY IN A MODEL OF INHIBITORY NEURONAL NETWORK.
}

\author{
Eleonora Catsigeras *
}

November 14th., 2008

\begin{abstract}
We analyze the dynamics of a deterministic model of inhibitory neuronal networks proving that the discontinuities of the Poincaré map produce a never empty chaotic set, while its continuity pieces produce stable orbits. We classify the systems in three types: the almost everywhere (a.e.) chaotic, the a.e. stable, and the combined systems. The a.e. stable are periodic and chaos appears as bifurcations. We prove that a.e. stable systems exhibit limit cycles, attracting a.e. the orbits.
\end{abstract}

Keywords: Chaotic sets, limit cycles, piecewise continuous dynamics, neuronal networks.

\section{INTRODUCTION}

We obtain rigorous mathematical results on the dynamics of a deterministic abstract discontinuous dynamical system, in a finite but large dimensional phase space. It comes from a non linear model of inhibitory neuronal networks, without delays, composed by equally or different $2 \leq n<+\infty$ pacemaker neurons, evolving according to an autonomous differential equation in the inter-spike interval times, and interacting among them by synaptical instantaneous currents in the spiking instants.

A vectorial autonomous differential equation governs the increasing potentials of the $n$ neurons as a function on time $t$, only during the inter-spike regime. On the other hand, the spiking regime holds when at least one neuron, say $i$, reaches a threshold level and gives a spike. Due to the synaptic connections among the neurons, this spike produces sudden changes in the potentials of the other neurons $j \neq i$ and resets the potential of the neuron $i$.

The synapsis is assumed to be inhibitory, i.e. phase redeeming, meaning that the potentials of the receiving neurons suffer negative changes in the spiking times. That is why the inhibitory synapsis is modeled by a matrix $\left\{H_{i j}\right\}_{i \neq j}, 1 \leq i, j \leq n$ of negative numbers $H_{i j}<0$ that represent the instantaneous discontinuity jumps in the potentials of the neurons $j \neq i$, produced by a spiking of the neuron $i$.

The autonomous differential equation, verifying some very wide assumptions, governs the system during the inter-spike intervals of time. It leads to a Poincaré map (Sotomayor [1979]), which is contractive, as we prove in Theorem 4 (see also Budelli et al.[1996]).

\footnotetext{
* Instituto de Matemática y Estadística Prof. Rafael Laguardia Facultad de Ingeniería, Universidad de la República. Uruguay.
} 
Finally, when synaptic inhibitory coupling is added, it produces asymptotic cyclic attractors in the phase space, whose existence we prove in Theorem 91). Nevertheless, the discontinuities of the synapsis also generate chaotic orbits, whose existence we prove in Theorem 7. While the limit cycles exhibit mostly asynchronous spikes, but in periodic patterns, they could seem irregular to an experimental observer, if their periods are very large, and be virtually mixed with the real chaotic orbits.

The model we study includes the networks of coupled leaky integrators and the relaxation oscillators. The mathematical approach, under particular hypothesis, on the integrate and fire and the relaxation oscillator models of many neurons was early analyzed by Mirollo \& Strogatz [1990] for homogeneous networks of excitatory neurons. Later Abbott \& van Vreeswijk [1993] generalized the results for non homogeneous systems, also with delay times and excitatory interactions. For strongly coupled networks of inhibitory or excitatory neurons Bessloff \& Coombes [2000] find synchronous and asynchronous behavior.

In the abstract dynamical system we define the complementary sets $C$ and $S$, of chaotic and stable future orbits respectively (Section 3).

The qualitative rigorous analysis, discrete-sizing the dynamics by means of a Poincaré map (see Sotomayor [1979]), was applied to homogeneous networks by Mirollo \& Strogatz [1990]. Also Budelli et al. [1991] and Catsigeras \& Budelli [1992] studied the asymptotic future behavior of inhomogeneous two neurons networks, with a Poincaré map in a codimension one section.

On the other hand discrete-sizings on time, are different mathematical approaches and can fit better with computer experiments, in which both time and space are discretesized. Cessac [2008] and Céssac \& Viéville [2008] obtain similar results to those in this paper, using different mathematical models. They also study discrete discontinuous neural networks. Their model, in spite of being similar in the evolution given by equation (1), is intrinsically different to consider the spike instants as predetermined by the observer.

In this paper we prove that the Poincaré map is discontinuous due to the inhibitory synapsis. Although it is piecewise contractive, we prove that its discontinuities play the role of chaos generators: due to them the chaotic set $C$ is never empty.

The abstract dynamical systems of this model are classified in three types according to their attractors a.e.. Calling $m$ to the Lebesgue probability measure, we define the a.e. chaotic systems (for which $C$ has full measure: $m(C)=1, m(S)=0$ ); the a.e. stable systems (for which $m(C)=0, m(S)=1$ ); and the combined systems (for which $m(C)>0, m(S)>0$ ). Generically, under some additional hypothesis, the systems are a.e. stable, and the chaotic set appears as a bifurcation among different stable systems. This kind of bifurcations were studied by Catsigeras, Rovella \& Budelli [2008], proving that they exhibit a chaotic Cantor set attractor.

In Theorem 9 of this paper we prove that the a.e. stable systems exhibit limit cycles attracting Lebesgue almost all the orbits, and due to them they are a.e. periodic. Nevertheless the period of the attracting limit cycles, and the number of them, are mostly determined by the relation among the inhibitory synaptic interactions. They can a priori be very large, and have few relation with the intrinsic individual periods of the integrate and fire neurons of the network. This last result fits with the numerical experiments in networks of $n$ different integrate and fire neurons. Postnova et al.[2007] analyzed the dynamics of computer simulated large networks, and obtained that the system can be 
driven through different synchronization states, but they are significantly different from the original periodic behavior of the individual cells.

\section{DEFINITIONS AND STATEMENTS.}

The phase space is a bounded cube $Q=[-\theta, \theta]^{n}$ of the vectorial space $\mathbb{R}^{n}$, whose points are the $n$-uples $V=\left(V_{1}, \ldots, V_{n}\right)$ with $-\theta \leq V_{i} \leq \theta, \forall 1 \leq i \leq n$. We denote

$$
I=\{1,2, \ldots, n\}
$$

to the set of neurons of the network. The variable $V_{i}$ is the potential states of the $i$-th. neuron of the network, and it evolves on time $t$.

In this model the synapses and the spikes of the neurons, can be produced at any instant $t_{i}$ : when the potential $V_{i}$ of at least one of the neurons (say the $i$-th neuron), reaches $\theta$ (the fixed threshold level). $t_{i}$ is not previously specified: it is the solution of the implicit function $\Phi_{i}^{t}=\theta$. We denote as $\Phi_{i}^{t}$ to the $i$-th. component of the vectorial flux solution $\Phi^{t}$ of an autonomous differential equation

$$
d V / d t=F(V), \forall V \in Q \subset \mathbb{R}^{n}, F: Q \mapsto \mathbb{R}^{n}, F \in \mathcal{C}^{1}
$$

This vectorial differential equation determines the potential $V_{i}$ of each neuron $i$ as a function on time $t$, only during the inter-spike regime. The solution $\Phi^{t}\left(V^{0}\right)$ depends on the initial state $V^{0}=\left(V_{1}^{0}, \ldots, V_{n}^{0}\right)$. We restrict to the case in which the system is composed by $n$ independent differential equations (i.e. $F_{i}$ depends only of $V_{i}$ ). Nevertheless the open conclusions we obtain (for instance the result in Theorem 44) holds even if the neurons slightly interact during the inter-spike intervals (i.e. $\partial F_{i} / \partial V_{j} \approx 0$ if $i \neq j$ ). We assume that

$$
F_{i}>0, \quad \partial F_{i} / \partial V_{i}<0 \quad \forall i \in I, \quad \partial F_{i} / \partial V_{j}=0 \forall j \neq i
$$

and $F_{i}$ and $\partial F_{i} / \partial V_{i}$ are bounded away from zero. The dynamics given by (1) and (2) is that of general pacemaker neurons, with strictly increasing potentials in dissipative regime, providing negative concavity of the free evolution on time, of the potential of each neuron in the inter-spike time intervals. This property leads to a contractive Poincaré map (Theorem 4) and produce, when synaptic inhibitory coupling is added, asymptotic cyclic attractors in the phase space (see Theorem 9), but also chaotic orbits (see Theorem 7). The limit cycles exhibit mostly asynchronous spikes, but in periodic patterns.

The model verifying (11) and (2) includes the networks of coupled leaky integrators and the relaxation oscillators, for instance if $F_{i}=-\gamma_{i}\left(V_{i}-\beta_{i}\right)$, with constant $0<\gamma_{i}, \beta_{i}$.

From an initial state $V^{0}$, the next spiking instant is

$$
\bar{t}\left(V^{0}\right)=\min _{1 \leq i \leq n} t_{i}\left(V^{0}\right)
$$

where $t_{i}\left(V^{0}\right)$ is the solution of the implicit function

$$
\Phi_{i}^{t}\left(V^{0}\right)=\theta \quad \Leftrightarrow \quad t=t_{i}\left(V^{0}\right)
$$

At instant $\bar{t}$ maybe more than one neuron reaches the threshold level $\theta$ simultaneously (although this occurs with zero Lebesgue probability in the initial state, if the system is 
inhibitory). We call $J\left(V^{0}\right)$ to that set of neurons that spike first, from the initial state $V^{0}$, i.e.

$$
J\left(V^{0}\right)=\left\{i \in I: t_{i}\left(V^{0}\right)=\bar{t}\left(V^{0}\right)\right\}
$$

Almost all initial states are such that $J\left(V^{0}\right)=\{i\}$ for a single neuron $i \in I$. The set $J$ of neurons gives spikes at time $\bar{t}$, i.e. their potentials reset to zero, and from the new initial state $W^{0}$ it restart the evolution according to the differential equation (11). Precisely

$$
V_{i}\left(\bar{t}^{-}\right)=\theta \Rightarrow V_{i}\left(\bar{t}^{+}\right)=W_{i}(0)=0 \quad \forall i \in J
$$

When spiking, each of the neurons $i \in J$, which are supposed to be inhibitory, produces a sudden synaptic current through its connections to the other neurons $j \notin J$. This synapsis produces a sudden change, of amplitude $-H_{i j}<0$, in the potential $V_{j}$. Precisely:

$$
V_{j}\left(\bar{t}^{-}\right)<\theta, \quad V_{j}\left(\bar{t}^{+}\right)=W_{j}(0)=V_{j}\left(\bar{t}^{-}\right)-\sum_{i \in J} H_{i j} \forall j \notin J
$$

\section{THE POINCARÉ SECTION AND THE FIRST RETURN MAP.}

In this section the dynamics of the first return map $\rho: B \mapsto B$ is adequately defined in a Poincaré section $B$ (see the definition of Poincaré section and map in Sotomayor [1979]). The section $B$ will be diffeomorphic to the union of a finite number of $n-1$ dimensional balls transverse to the flux.

In the compact phase space $Q=\left\{V \in \mathbb{R}^{n}:-\theta \leq V_{i} \leq \theta \forall i \in I\right\}$ we take the following $n$-1-dimensional section:

$$
B=\bigcup_{k=1}^{n} \widehat{B}_{k} \quad \text { where } \widehat{B}_{k}=\left\{V \in Q: V_{k}=0\right\}
$$

Its topology is that induced by $\widehat{B}_{k} \subset \mathbb{R}^{n-1}$.

We assert that $B$ is transversal to the flux $\Phi^{t}$.

Proof: It is enough to prove that $\widehat{B}_{k}$ is transversal to the flux for all $k=1, \ldots, n$. This last assertion is deduced from the property $d \Phi_{i}^{t} / d t=F_{i}\left(\Phi^{t}\right)>0$, due to (2), as follows: the $n$ components of the vector $d \Phi^{t} / d t$, which is tangent to the flux, are positive, in particular its $k$-th. component. On the other hand, the manifold $\widehat{B}_{k}$ is $n-1$ dimensional, defined by the equation $V_{k}=0$. Therefore its tangent subspace $S_{k} \sim \mathbb{R}^{n-1}$ is formed by all the vectors in $\mathbb{R}^{n}$ such that have null their $k$-th. component. Then we deduce:

$$
S_{k} \oplus\left[\frac{d \Phi^{t}}{d t}\right]=\mathbb{R}^{n}
$$

where $[\vec{U}]$ denotes the subspace generated by the vector $\vec{U} \in \mathbb{R}^{n}$.

Due to the definition of the mathematical spike in equation (6), from any initial state $V^{0} \in Q$ the system arrives to the Poincaré section $B$ in a finite time $\bar{t}\left(V^{0}\right)$ given by equations (3) and (4). So in particular for $V^{0} \in B$, we have defined the first return Poincaré map $\rho$ :

$$
\rho: B \mapsto B, \quad \rho\left(V^{0}\right)=W^{0}=V^{\bar{t}^{+}\left(V^{0}\right)}
$$


The sequence of inter-spike intervals (ISI) is given by the evolution of the system through the iterates $\rho^{p}, p=1,2, \ldots$ of the return map $\rho$. The ISI sequence is

$$
\bar{t}\left(V^{0}\right), \bar{t}\left(\rho\left(V^{0}\right)\right), \bar{t}\left(\rho^{2}\left(V^{0}\right)\right), \ldots, \bar{t}\left(\rho^{p}\left(V^{0}\right)\right), \ldots
$$

If the orbits of the discrete dynamical system by iteration of $\rho$ are attracted to limit cycles or not, the ISI sequence will be finally periodic or not. If the dynamics of $\rho$ exhibits chaotic attractors, then the ISI sequence also. Even in the periodic case, its period depends of the map $\rho$, (and also of the initial state if there were many limit cycles). But $\rho$ depends strongly of the matrix of synaptic interactions $H_{i j}$. Therefore, the network composed by the inhibitory coupling of $n$ oscillators, may have a dynamics which widely differs from the behavior of each isolated neuron.

We iterate the Poincaré map $\rho\left(V^{0}\right)=W^{0}$, and after each iterate we reset the time to consider the solution $\Phi^{t}\left(W^{0}\right)$ of the equation (1), from the new initial state $W^{0} \in B$. To simplify the notation we will omit the supraindex ${ }^{0}$ : from now $U, V, W$ denote points in the Poincaré section.

From equalities (6), (7) and (9) we have the following formula for the Poincaré map:

$$
\begin{array}{cl}
\rho_{j}(V)=\Phi_{j}^{\bar{t}(V)}(V)-\sum_{i \in J(V)} H_{i j} & \forall j \notin J(V) \\
\rho_{i}(V)=0 & \forall i \in J(V)
\end{array}
$$

Denote $\wp(I)$ to the family of all non empty parts of the set $I$ of neurons. Roughly speaking $\wp(I)$ the collection of all possible "words" of different neurons, of any length $\geq 1, \leq n$, without considering the order. For each $J \in \wp(I)$ (say for instance, $J=\{1,2\}$ ), define:

$$
B_{J}=\{V \in B: \quad J(V)=J\}
$$

In the example $J=\{1,2\}$, the set $B_{\{1,2\}} \subset B$ is composed by all the initial states in the Poincaré section $B$ for which neurons 1 and 2 , and only them, will arrive first and simultaneously to the threshold level.

Remark 1 Consider the difference, not only in notation but in significance, among the sets $B_{\{i\}}$ and $\widehat{B}_{i}$.

On one hand, $B_{\{i\}}$, for a fixed index $i=1,2, \ldots, n$, is the set of all the initial states in the Poincaré section $B$, from which neuron $i$, and only neuron $i$, will arrive first to the threshold level, after the system's evolution in the inter-spike regime. Then $B_{\{i\}} \cap B_{\{j\}}=\emptyset$ if $i \neq j$. Also $\cup_{i=1}^{n} B_{\{i\}} \subset \neq B=\cup_{J \in \cap P(I)} B_{J}$.

On the other hand, according to its definition in (8), $\widehat{B}_{k}$, for a fixed index $k=1,2, \ldots, n$, is the set of all the states in the Poincaré section $B$, in which the potential level $V_{k}$ of the neuron $k$ is the reset level zero. We have $\widehat{B}_{k} \cap \widehat{B}_{h}=\left\{V \in B: V_{h}=V_{k}=0\right\} \neq \emptyset \quad \forall h \neq k$, and $B=\cup_{k=1}^{n} \widehat{B}_{k}$. Also, due to the definition of the Poincaré map $\rho$ in the equality after (10), we deduce that $\rho\left(B_{\{i\}}\right) \subset \widehat{B}_{i}, \quad \rho\left(B_{\{i, j\}}\right) \subset \widehat{B}_{i} \cap \widehat{B}_{j}$.

Remark 2 Due to formulae (10) the Poincaré map $\rho$ is continuous in each piece $B_{J}$, in particular in $B_{\{i\}}$ for all $i \in I$. The set $B_{J}$, for each $J \in \wp(I)$, is called a continuity piece of $\rho$.

From (10), due to the transversality of the flux $\Phi^{t}$ to $\widehat{B}_{i}, \rho$ transforms homeomorphically $B_{\{i\}} \cap \widehat{B}_{k}$ onto its image in $\widehat{B}_{i}$. So it is an open map. Nevertheless it is not globally injective because each point may have different pre-images in $\widehat{B}_{k}$ and $\widehat{B}_{h}$ for $h \neq k$. 
The partition $\mathcal{P}$ and the set $\partial \mathcal{P}$ of discontinuities.

By construction, for different subsets $J^{1} \neq J^{2} \in \wp(I): B_{J_{1}} \cap B_{J_{2}}=\emptyset$. Any initial state $V$ in $B$ belongs to one and only one set $B_{J}$ (exactly to that $B_{J}$ such that $J(V)=J$ ). Therefore:

The collection $\left\{B_{J}\right\}_{J \in \wp(I)}$ form a partition $\mathcal{P}$ of the Poincaré section $B$ into the continuity pieces of the return map $\rho$.

(3) and (4) implies that the set of $V \in B$ such that $\bar{t}(V)=t_{i}(V)<t_{j}(V) \forall j \neq i$ is open. In other words it is open the set $B_{\{i\}}$ (i.e. the set of initial states from where the neuron $i$, and only the neuron $i$, arrives first to the threshold level). On the other hand $B_{J}$ has empty interior if $\# J \geq 2$.

We consider only those not dead neurons (i.e. they do not remain under the threshold level forever) and rename as $I$ to that set of neurons. If there is only one, then there is not interactive network to study. So let us suppose that $\# I \geq 2$ and then $\left\{B_{i} \neq \emptyset: \quad i \in I\right\}$ is a collection of at least two open and non empty sets. As the space $B$ is connected, we conclude that the topological frontiers of the pieces of the partition $\mathcal{P}$ is not empty. Define:

$$
\partial \mathcal{P}=B \backslash \bigcup_{i=1}^{n} B_{\{i\}}=\bigcup_{\# J \geq 2} B_{J}=\bigcup_{i=1}^{n} \partial B_{\{i\}}
$$

\section{Topological properties of the Poincaré map.}

We use the following norm to compute the distances in each $\widehat{B}_{k} \subset B$, for $k \in I$ :

$$
\|V-W\|=\max _{i \in I}\left|V_{i}-W_{i}\right| \quad \forall V, W \in \widehat{B}_{k}
$$

Assume the following generic hypothesis in the parameters of the system:

$$
H_{i j} \neq \theta \quad \forall i \neq j \in I
$$

Define the expansivity constant:

$$
\alpha=\frac{\min _{i \neq j}\left|\theta-H_{i j}\right|}{4}>0
$$

Lemma 3 If $V \in \partial \mathcal{P}$ then the Poincaré map $\rho$ is discontinuous in $V$ and the discontinuity jumps in $V$ are larger than $3 \alpha$. Precisely, there exists a sequence of points $U^{m} \rightarrow V$ such that $\lim _{m \rightarrow+\infty}\left\|\rho\left(U^{m}\right)-\rho(V)\right\|>3 \alpha$

Proof: If $V \in \partial \mathcal{P}$ then $V \in B_{J}$ for some $J \in \wp(I), \# J \geq 2$. There exist $i \neq j$ in $J$ and therefore $B_{J} \subset \partial B_{\{i\}}$. By the definition of topological frontier, there exists a sequence of points $U^{m} \rightarrow V$ such that $U^{m} \in B_{\{i\}}$.

$$
\rho_{j}\left(U^{m}\right)=\Phi_{j}^{\bar{t}\left(U^{m}\right)}\left(U^{m}\right)-H_{i j} \rightarrow_{m \rightarrow+\infty} \Phi_{j}^{\bar{t}(V)}(V)-H_{i j}
$$

The last limit is computed recalling that the solution $\Phi^{t}$ of the differential equation (11) depends continuously on the initial state $V$, and the ISI time $\bar{t}(V)$, defined as the minimum 
in equality (3) of the solutions of the implicit equations (44), also depends continuously on $V$, due to the Implicit Function Theorem. (Rey Pastor \& col. [1968]).

But $j \in J, V \in B_{J} \Rightarrow j \in J(V)$, or in other terms, $V$ is an initial state for which the neuron $j$ arrives to the threshold level. Then $\Phi_{j}^{\bar{t}(V)}(V)=\theta$, and by equalities (10), $j \in J(V) \Rightarrow \rho_{j}(V)=0$. Then:

$$
\begin{aligned}
\lim \rho_{j}\left(U^{m}\right) & =\theta-H_{i j}, \quad \rho_{j}(V)=0 \\
\left|\lim \rho_{j}\left(U^{m}\right)-\rho_{j}(V)\right| & =\left|\theta-H_{i j}\right| \geq 4 \alpha>3 \alpha
\end{aligned}
$$

By the definition of limit, for all $m$ large enough we obtain $\left\|\rho_{j}\left(U^{m}\right)-\rho_{j}(V)\right\|>3 \alpha$.

The Lemma 3 explains why the discontinuities of $\rho$ act as saddle type orbits in continuous systems, to produce expansion and chaos. The points of $\partial \mathcal{P}$ act on their neighborhoods with an infinite rate of expansion in some directions, given by instantaneous expansivity larger than an uniform number $3 \alpha>0$. But they also have directions of contraction, as they are in the frontier of the open pieces $B_{\{i\}}$ of continuity of $\rho$, where the following result holds:

Theorem 4 The Poincaré map $\rho: B \mapsto B$ is uniformly contractive in each of its continuity pieces $B_{\{i\}} \cap \rho(B)$. Precisely, there exists $0<\lambda<1$ and a distance dist $t_{c}$ such that, if $V, U \in B_{\{i\}} \cap \rho(B)$ for some $i \in I$, then

$$
\operatorname{dist}_{c}(\rho(V), \rho(U)) \leq \lambda \operatorname{dist}_{c}(V, U)
$$

Besides, there exists $K>0$ such that for all $V, U \in B$ :

$$
\frac{1}{K}\|V-U\| \leq \operatorname{dist}_{c}(V, U) \leq K\|V-U\|
$$

We note that the distance $\operatorname{dist}_{c}$ is not necessarily the norm $\|\cdot\|$ defined in (11). Nevertheless, they are equivalent in $\mathbb{R}^{n-1}$. For simplicity we will omit the not zero constant factors $1 / K$ and $K$ in the forward computations along this work, and use $\|\cdot\|$ as if it were dist $_{c}$. This is not a restriction because, as a consequence of Theorem 4 for any norm there exists an iterate $p_{0}$ such that $\rho^{p_{0}}$ is contractive in its continuities pieces: $\left\|\rho^{p}(V)-\rho^{p}(U)\right\| \leq$ $K \operatorname{dist}_{c}\left(\rho^{p}(V), \rho^{p}(U)\right) \leq K \lambda^{p} \operatorname{dist}_{c}(V, U) \leq K^{2} \lambda^{p}\|V-U\| \leq(1 / 2)\|V-U\|$ for all $p$ large enough.

Proof: Define $0<\epsilon_{0}=\min _{h \neq j} H_{h j}$. Apply equality (10) and use, at the end of the following formulae, the Lagrange Mean Value Theorem (Rey Pastor \& col. [1968]), to compute the difference of $\Phi_{i}^{t}(V)$, for different values of the time $t$, as the increment $\Delta t$ multiplied by the derivative in an intermediate time $T^{m}$ :

$$
\begin{gathered}
V \in \rho\left(B_{\{k\}}\right) \Rightarrow V_{k}=0, \quad V_{j} \leq \theta-\epsilon_{0} \forall j \in I \\
V \in \widehat{B}_{k}=\left\{V \in B: V_{k}=0\right\} \\
V \in B_{\{i\}} \Rightarrow \phi_{i}^{t}(V)(V)=\theta, \quad \phi_{i}^{0}(V)=V_{i} \\
\epsilon_{0} \leq \theta-V_{i}=\bar{t}(V) d \Phi_{i}^{t}(V) /\left.d t\right|_{t=T^{m}} \\
\epsilon_{0} \leq \bar{t}(V) F_{i}\left(\Phi^{T^{m}}(V)\right) \\
\left.\Rightarrow \bar{t}(V) \geq \epsilon_{0} / \max _{1 \leq i \leq n} \max _{V \in Q} F_{i}(V)\right)=t_{0}>0
\end{gathered}
$$


By the Tubular Flux Theorem (Sotomayor [1979]), there exists a $\mathcal{C}^{1}$ bounded diffeomorphism which is a spatial change of variables $\xi: V \mapsto \widehat{V}$ from $Q \subset \mathbb{R}^{n}$ onto $\widehat{Q} \subset \mathbb{R}^{n}$, such that $\left.\xi\right|_{B}=i d$ and the solutions of the differential equation (1) in $Q$ verify $d \widehat{V} / d t=\vec{a}$ in $\widehat{Q}$, where $\vec{a} \in \mathbb{R}^{n}$ is a constant vector with positive components. It verifies: $\xi\left(\phi^{t}(V)\right)=$ $\xi(V)+\vec{a} \cdot t, d \xi F(V)=\vec{a} \forall V \in Q$.

Define in $\mathbb{R}^{n}$ the orthogonal projection $\pi$ onto the $(n-1)$-dimensional subspace $a_{1} V_{1}+$ $a_{2} V_{2}+\ldots+a_{n} V_{n}=0$. The flux of the differential equation (11), after the change $\xi$ of variables in the space, is ortogonal to that subspace, and is transversal to $\widehat{B}_{k}$. Consider any real function $g$ :

$$
\begin{array}{r}
\forall \widehat{V}, \widehat{V}+d \widehat{V} \in \mathbb{R}^{n}: \\
\pi(d \widehat{V})=\pi(d \widehat{V}+\vec{a} g(\widehat{V}, d \widehat{V})) . \\
\forall V, V+d V, \quad U \in \widehat{B}_{k}, \text { define } \\
\operatorname{dist}_{c}(V, V+d V)=\left\|\pi\left(d \xi_{V} d V\right)\right\| \\
\operatorname{dist}_{c}(V, U)=\int_{0}^{1}\left\|\pi \cdot d \xi_{V+t(U-V)} \cdot(U-V)\right\| d t
\end{array}
$$

As $\xi$ is a $\mathcal{C}^{1}$ diffeomorphism, its derivative and the derivatives of its inverse, are bounded in the compact set $B$, and so the distance dist $_{c}$ defined above verifies the last thesis of this Theorem. It is left to prove that $\rho$ is contractive with this distance in $B_{\{i\}} \cap \widehat{B}_{k}$.

Let us apply $\rho$ to $V, V+d V \in B_{\{i\}} \cap \widehat{B}_{k}$. We use the equality (10) with $J(V)=\{i\}$, in which for convenience we agree to define $H_{i i}=\theta$. We shall use the derivation formula of the flux of the differential equation respect to its initial state:

$$
\begin{gathered}
\left.\partial \Phi_{j}^{t}\left(V_{j}\right) / \partial V_{j}=\exp \left(\int_{0}^{t}\left(\partial F_{j} / \partial V_{j}\right)\left(\Phi_{j}^{s}\left(V_{j}\right)\right) d s\right)\right) \\
\text { Define: } \quad-\gamma=\max _{1 \leq j} \max _{V \in Q} \partial F_{j}\left(V_{j}\right) / \partial V_{j} \quad<0
\end{gathered}
$$

In what follows $i$ is fixed. It is the value of the index of the continuity piece $B_{\{i\}}$ given in the hypothesis of this lemma. On the other hand, $j=1,2, \ldots, i, \ldots, n$ is the index of the general component $\partial \Phi_{j}^{t} / \partial t$ of the tangent vector of the flux $\Phi^{t}$, and of the general component $\rho_{j}(V)$ of the Poincaré map whose derivative we are computing. We must compute all their components, so we must include the case in which $j=i$.

The formula (10) and the inequality (13) lead to:

$$
\begin{gathered}
\rho(V)-\rho(V+d V)=d \rho(V) \cdot d V=\left[\left(\partial \rho_{j}(V) / \partial V_{j}\right) d V_{j}+\left(\partial \rho_{j}(V) / \partial V_{i}\right) d V_{i}\right]_{1 \leq j \leq n} \partial \rho_{j}(V) / \partial V_{j} \\
\left.=\left.\left(\partial \Phi_{j}^{t}\left(V_{j}\right) / \partial V_{j}\right)\right|_{t=\bar{t}(V)}=\exp \left(\int_{0}^{\bar{t}(V)}\left(\partial F_{j} / \partial V_{j}\right)\left(\Phi_{j}^{s}\left(V_{j}\right)\right) d s\right)\right) \leq e^{-\gamma t_{0}} \partial \rho_{j}(V) / \partial V_{i}= \\
=\left.\left(d \Phi_{j}^{t}\left(V_{j}\right) / d t\right)\right|_{t=\bar{t}(V)} \cdot\left(d \bar{t}\left(V_{i}\right) / d V_{i}\right)=g(V) \cdot F_{j}\left(\Phi^{\bar{t}(V)}(V)\right),
\end{gathered}
$$

where $g(V)=d \bar{t}\left(V_{i}\right) / d V_{i}$ is the real function obtained deriving respect to $V_{i}$ the implicit equation $\theta=\Phi_{i}^{\bar{t}\left(V_{i}\right)}\left(V_{i}\right)$. Call $\vec{e}_{j}$ to the $j$-th. vector of the canonic base in $\mathbb{R}^{n}$ and join all the results above:

$$
\pi \cdot d \xi_{\rho(V)} \cdot(\rho(V)-\rho(V+d V))=\pi \cdot d \xi_{\rho(V)} \cdot d \rho(V) \cdot d V=
$$




$$
\begin{gathered}
=\pi \cdot d \xi_{\rho(V)} \cdot\left(\sum_{j=1}^{n}\left(\partial \rho_{j}(V) / \partial V_{j}\right) \cdot d V_{j} \vec{e}_{j}\right)+\pi \cdot d \xi_{\rho(V)} \cdot\left(g(V) \cdot F\left(\phi^{\bar{t}(V)}(V)\right)=\right. \\
\left.=\pi \cdot d \xi_{\rho(V)} \cdot\left(\sum_{j=1}^{n}\left(\partial \rho_{j}(V) / \partial V_{j}\right) \cdot d V_{j} \vec{e}_{j}\right)+g(V) \cdot \pi \cdot d \xi_{\rho(V)} \cdot F\left(\phi^{\bar{t}(V)}(V)\right)\right)= \\
=\pi \cdot d \xi_{\rho(V)} \cdot\left(\sum_{j=1}^{n}\left(\partial \rho_{j}(V) / \partial V_{j}\right) \cdot d V_{j} \vec{e}_{j}\right)+\pi(g(V) \cdot \vec{a})= \\
=\pi \cdot d \xi_{\rho(V)} \cdot\left(\sum_{j=1}^{n}\left(\partial \rho_{j}(V) / \partial V_{j}\right) \cdot d V_{j} \vec{e}_{j}\right)
\end{gathered}
$$

Now we define the number $0<\lambda=e^{-\gamma t_{0}}<1$ and observe from the computations above that:

$$
0<\partial \rho(V) / \partial V_{j} \leq e^{-\gamma t_{0}}=\lambda<1 .
$$

Applying the definition of the differential distance dist $_{c}$ in (14), and the equality (15), we obtain:

$$
\begin{gathered}
\operatorname{dist}_{c}(\rho(V), \rho(V+d V))=\left\|\pi\left(d \xi_{\rho(V)} d \rho_{V} \cdot d V\right)\right\| \leq \\
\lambda\|\pi d \xi \cdot d V\|=\lambda \operatorname{dist}_{c}(V, V+d V)=\lambda\left\|\pi\left(d \xi_{V} d V\right)\right\|
\end{gathered}
$$

Integrating by formula (14) we conclude:

$$
\operatorname{dist}_{c}(\rho(V), \rho(U)) \leq \lambda \operatorname{dist}_{c}(V, U)
$$

\section{Measure properties of the Poincaré map.}

Let $m$ be the $(n-1)$-dimensional Lebesgue probability measure in the Poincaré section $B$. Let us prove that

$$
m(\partial \mathcal{P})=m\left(\bigcup_{\# J \geq 2} B_{J}\right)=0
$$

In fact, each $B_{J}$, if $\# J \geq 2$, is the finite union of $C_{2}^{n} n-(\# J)$-dimensional manifolds in $B$, obtained when, for two or more different values of $i \in I$, the respective solutions $t_{i}(V)$ of the implicit equations (4), are equal.

Our aim is to study the attractors. The weakest condition required to a set $A \subset B$ to be an attractor is that its basin of attraction has positive Lebesgue measure. So, we may take out the points of the measure zero set $\partial \mathcal{P}$.

\section{The set $B^{\prime}$ of the points with infinite itinerary.}

Define

$B^{\prime}=\left\{V \in B: \rho^{j}(V) \notin \partial \mathcal{P} \quad \forall j \geq 0\right\} \subset B \backslash \partial \mathcal{P}$

$B_{\{i\}} \cap B_{\{j\}}=\emptyset$ if $i \neq j$ and $\bigcup_{i \in I} B_{\{i\}}=B \backslash \partial \mathcal{P} \Rightarrow$

$B^{\prime}=\bigcap_{p=0}^{+\infty} \rho^{-p}(B \backslash \partial \mathcal{P}), \quad B \backslash B^{\prime}=\bigcup_{p=0}^{+\infty} \rho^{-p}(\partial \mathcal{P})$.

The following assertion characterizes the set $B^{\prime}$ :

$V \in B^{\prime} \Leftrightarrow \exists$ a unique sequence $\left\{i_{p}\right\}_{p \geq 0}$ such that $\rho^{p}(V) \in B_{\left\{i_{p}\right\}} \forall p \geq 0$. 
This sequence is called the itinerary of $V$, and it is the infinite sequence of neurons that will spike (in different times), in the order they reach the threshold potential, from the initial state $V$.

$\rho$ is continuous in each $B_{\{i\}}$. Therefore, for all $p \geq 0$ and for all $V \in B^{\prime}$, the iterate map $\rho^{p}$ is continuous in $V$. As $B^{\prime}$ is the numerable intersection of open and dense sets, it is dense.

Theorem 5 The set $B^{\prime}$ with infinite itinerary has full Lebesgue measure in $B$, i.e: $m(B \backslash$ $\left.B^{\prime}\right)=0$.

Proof: To prove that $m\left(\rho^{-1}(\partial \mathcal{P})\right)=0$, once it is known that $m(\partial \mathcal{P})=0$, use the same argument as to prove by induction that for all $p \geq 1: m\left(\rho^{-p}(\partial \mathcal{P})\right)=0$, once it is known for $p-1$.

In fact, we apply Liouville Formula (Sotomayor [1979]) to compute the following Lebesgue integral:

$$
\begin{aligned}
0=m(\partial \mathcal{P}) & \left.\geq m\left(\rho^{-1}(\partial \mathcal{P})\right)\right)= \\
& =\int_{V \in \rho^{-1}(\partial \mathcal{P})}|\operatorname{det}(D \rho)(V)| d m(V)
\end{aligned}
$$

and conclude that $m\left(\left(\rho^{-1}(\partial \mathcal{P})\right) \cap\{V \in B ;|\operatorname{det}(D \rho)|>0\}\right)=0$. To prove that $m\left(\rho^{-1}(\partial \mathcal{P})\right)=$ 0 it is enough to prove that the Jacobian $\operatorname{det}(D \rho) \neq 0$ a.e. in $B$.

To apply the Liouville Formula we shall first prove that $\rho$, given by formulas (10), is differentiable a.e. in the integration set. The first technical problem arises because $\rho$ is not differentiable in all the points: it is neither in the points of discontinuity $\partial \mathcal{P}$ nor in the points of $\bigcup_{h \neq k \in I}\left(\widehat{B}_{h} \cap \widehat{B}_{k}\right)$. In fact, in these last set the Poincaré section itself fails to be a local differentiable manifold. The set of those exceptional points has zero Lebesgue measure because they are contained in $(n-2)$-dimensional subspaces.

The second technical difficulty is to check that $\operatorname{det} D \rho \neq 0$ a.e. The derivative of the Poincaré map can be computed directly from formulas (10), in each continuity piece $B_{\{i\}}$ intersected with each $\widehat{B}_{k} \backslash \bigcup_{h \neq k} \widehat{B}_{h}$. In those sets $B$ is a local differentiable manifold. Computations in (??) lead to

$\operatorname{det} D \rho(V)=\left[\prod_{j \neq i, j \neq k}\left(\partial \rho_{j}(V) / \partial V_{j}\right)\right] \cdot\left(\partial \rho_{k}(V) / \partial V_{i}\right)$ All these factors are computed in equalities (??) and are not zero due to hypothesis (2), so det $D \rho \neq 0$ a.e.

\section{CHAOTIC AND STABLE SETS.}

We will divide the Poincaré section $B$ in two complementary sets $S$, formed by stable orbits, and $C=B \backslash S$, the chaotic ones, according to Definition 6. The set $S$ is formed by stable future orbits under any uniform sufficiently small perturbation that can be added at any step of the iteration of $\rho$. In the set $C=B \backslash S$ there are arbitrarily small perturbations that, if added in some step of the iteration of $\rho$, drastically change the future orbits and their asymptotic behavior. To have a criteria of chaos, what we call drastic changes in the phase state, we consider the expansivity constant $\alpha>0$ defined in (12).

Definition 6 Stable and chaotic sets. $V \in B$ is stable if there exists $\delta>0$ such that $\forall p \geq 0, \quad \forall W \in B$, if $\left\|\rho^{p}(V)-W\right\| \leq \delta$ then $\left\|\rho^{k}\left(\rho^{p}(V)\right)-\rho^{k}(W)\right\| \leq \alpha \forall k \geq 1$. 
$V \in B$ is chaotic if it is not stable. The opposite of the definition of stable point holds as follows: $V \in B$ is chaotic if and only if for all $\delta>0$ there exists $p \geq 0$, and there exists $W \in B$ such that $\left\|\rho^{p}(V)-W\right\| \leq \delta$ and $\left\|\rho^{k}\left(\rho^{p}(V)\right)-\rho^{k}(W)\right\|>\alpha$ for some $k \geq 1$. $S$ is the set of all the stable orbits and $C=B \backslash S$ is the set of all the chaotic orbits.

It is immediate from the definitions above that $S$ is forward invariant: $\rho(S) \subset S$, and thus, its complement $C$ is backward invariant: $\rho^{-1}(C) \subset C$.

Given $\delta>0$ fixed, we define the uniform stable set $S_{\delta} \subset S$ as the set of $V \in B$ such that $\forall p \geq 0, \quad \forall W \in B, \quad$ if $\left\|\rho^{p}(V)-W\right\| \leq \delta \quad$ (with $\delta$ previously fixed), then $\| \rho^{k}\left(\rho^{p}(V)\right)-$ $\rho^{k}(W) \| \leq \alpha \forall k \geq 1$. From the definitions above observe that $\rho\left(S_{\delta}\right) \subset S_{\delta}$ and that any point in $S$ is in $S_{\delta}$ for some $\delta>0$. Therefore, to study the dynamics of the stable points, it is enough to study the dynamics in the sets $S_{\delta}$.

Theorem 7 The set $C$ of chaotic points is never empty. Precisely: $C \supset B \backslash B^{\prime} \supset \partial \mathcal{P} \neq \emptyset$, where $B^{\prime}$ is the set of points in the Poincaré section $B$ with infinite itinerary, and $\partial \mathcal{P}$ is the set of discontinuities of $\rho$.

Even more, if $V \in \partial \mathcal{P}$ then for all $\delta>0$ there exists $W \in B$ such that $\|V-W\|<$ $\delta,\|\rho(V)-\rho(W)\|>3 \alpha$, where $\alpha$ is the expansivity constant of the system.

Proof: From the note at the end of Remark 2 and from the definition of the set $B^{\prime}$ of the points with infinite itinerary, we get $\emptyset \neq \partial \mathcal{P} \subset B \backslash B^{\prime}$.

First, let $V \in \partial \mathcal{P}$. We shall prove that $V \in C$. From Lemma 3 there exists sequence of points $U^{m} \rightarrow V$ such that $\lim \left\|\rho\left(U^{m}\right)-\rho(V)\right\|>3 \alpha$. Given $\delta>0$ for all $m \geq 1$ large enough the points $U^{m} \rightarrow V$ verify $\left\|U^{m}-V\right\|<\delta$. Therefore, taking $W=U^{m}$ the point $V$ verifies the definition of chaotic point with $p=0$ and $k=1$.

Second, take $V \in B \backslash B^{\prime}=\bigcup_{p \geq 0} \rho^{-p}(\partial \mathcal{P})$. We shall prove that $V \in C$. We know that for some $p \geq 0 \rho^{p}(V) \in \partial \mathcal{P}$. In the first step we proved $\rho^{p}(V) \in C$, so $V \in \rho^{-p}(C) \subset C$, because $C$ is backward invariant.

\section{Classification of systems.}

- a.e.stable systems: $m(S)=1$ and $m(C)=0$. In Theorem 9 we prove that the a.e the limit set is composed only by periodic sinks (limit cycles). Nevertheless the set $C$ of chaotic points in not empty as proved in Theorem 7.

- Chaotic systems: $m(C)=1$ and $m(S)=0$. By definition the limit set $A$ of $C$ is a chaotic attractor. In Catsigeras \& al. [2008] it is proved that $A$ is a Cantor set attractor. - Partially chaotic and partially periodic systems: $m(C)>0$ and $m(S)>0$. We will show that the points in $S$ are attracted to limit cycles.

Remark: We did not find $\mathcal{C}^{1}$ examples in our model of systems for which $m(C)>0$ although due to Theorem 7 the set $C$ of chaotic points is never empty. Nevertheless it is possible (but not immediate) to construct piecewise continuous $\mathcal{C}^{0}$ maps $\rho$ in a $n-1 \geq 2$ compact ball $B$, that are uniformly contractive in each of their continuity pieces and such that $C=B, S=\emptyset$.

Some known results.

Suppose allowed any small perturbation of the system structure, such that, instead of having the differential vectorial equation (11), we have $d V / d t=G(V)$ with $\|G-V\|_{\mathcal{C}^{1}}<\epsilon$ for some $\epsilon>0$ sufficiently small. By continuity in the $\mathcal{C}^{1}$ topology of the functional 
space, the first two assumptions (2) are also verified by $G$ and the third one transforms in $\partial G_{i} / \partial V_{j} \approx 0 \quad \forall j \neq i$.

Besides, instead of restricting to a constant matrix $H_{0}=\left[H_{i j}\right]_{i, j \in I}$ to describe the synaptic interactions, allow a matrix $-H(V)$ of negative numbers $-H(\cdot)<0$ to be a $\mathcal{C}^{1}$ function such that $\left\|H-H_{0}\right\|_{\mathcal{C}^{1}}<\varepsilon$.

The new Poincaré map $\rho$ will move respect to the old one. The new set of discontinuities $\partial \mathcal{P}$ of the Poincaré map will be (with the Hausdorff distance) near the old one, because its points are those defined by implicit functions, whose equations have $\mathcal{C}^{1}$ dependence on $G, H \in \mathcal{C}^{1}$. So they are near the old ones if $\|G-F\|_{\mathcal{C}^{1}}$ and $\left\|H-H_{0}\right\|_{\mathcal{C}^{1}}$ are small enough. We conclude that, if $\varepsilon>0$ is sufficiently small, the new Poincaré map will be $\mathcal{C}^{1}$ near the old one, in each of its continuity pieces.

In this scenario, the following results are known, leading to the generality of the property $m(S)=1, m(C)=0$.

- If provided the additional open hypothesis of separation (pairwise disjointness of the closure of the images of the different pieces of continuity for some iterate of $\rho$ ), then Catsigeras \& al.[2008] proved that generically (open and dense in the $\mathcal{C}^{0}$ topology), there exist a finite number of limit cycles that attracts all the orbits of $B^{\prime}$. We conclude that $S=B^{\prime}$, and thus $m(S)=1$.

- If the contractive map $\rho$ is affine in each of its continuity pieces, then Cessac [2008] proved that for generic values of the real parameters, not only in the topological sense (open and dense family of systems), but also in the Lebesgue measure sense (in the space of finite number of the real affinity parameters), there exists at least one and up to a finite number of limit cycles, which attract all the orbits of $B^{\prime}$. We conclude that $S=B^{\prime}$ and $m(S)=1$.

- If the contractive map $\rho$ is not affine, but the matrix of interactions $H$ is constant, Cessac \& Viéville [2008] proved that either there exists limit cycles attracting the points of $B^{\prime}$ or the dynamics has positive entropy.

\section{Definition 8 Omega limit and limit cycles.}

For any $V \in B$, its omega-limit $\omega(V) \in B$ is the set of limit points of the future orbit of $V$. Precisely:

$$
\omega(V)=\left\{W \in B: \exists p_{j} \rightarrow+\infty \text { such that } \rho^{p_{j}}(V) \rightarrow W\right\}
$$

As the phase space $B$ is compact, the omega limit set of any point is not empty. The Poincaré map $\rho$ is not continuous, so $\omega(V)$ is not necessarily forward invariant. Nevertheless the omega limit set is the same for all the points in the same orbit, as it is easy to check.

A set $L \in B$ is a limit cycle (also called a periodic sink) if it is a single periodic orbit and its basin attraction $B(L)$ contains an open neighborhood of $L$, being:

$$
\begin{gathered}
L=\left\{V, \rho(V), \ldots, \rho^{r-1}(V)\right\}, \quad \rho^{r}(V)=V \quad r \geq 1 \\
B(L)=\{W \in B: \omega(W)=L\}
\end{gathered}
$$

Theorem 9 If for some $\delta>0$ the set $S_{\delta}$ of uniform stable points is not empty then, there exists a finite number $N$ of limit cycles $L_{1}, \ldots, L_{N}$ such that the union $\bigcup_{k=1}^{N} B\left(L_{k}\right)$ of their basins of attractions includes $S_{\delta}$. 
Remark: Let us show the main consequence of this theorem: if the set $S$ of stable points has positive measure, it is not empty and, because all its points are included in uniformly stable sets $S_{\delta}$, they all are attracted to limit cycles. By definition of limit cycles, the basins of attraction of different limit cycles are pairwise disjoint containing open sets. The topology in $B$ has numerable basis, so we conclude that there are at most a numerable quantity of limit cycles attracting all the orbits of $S$. If besides $m(S)=1$, then a.e. point in $B$ is attracted to a limit cycle.

We will prove Theorem 9 at the end of the next Section.

\section{THE ATOMIZATION OF THE SPACE.}

Let us consider a system for which the stable set $S$ is not empty. All the results in this Section will hold under this hypothesis.

As observed at the end of Definition [6: $S \neq \emptyset \Rightarrow S_{\delta} \neq \emptyset$ for some $\delta>0$, and thus also for all sufficiently small $\delta>0$. Fix $0<\delta<\alpha$ such that $S_{\delta} \neq \emptyset$, where $\alpha$ is the expansivity constant. We recall that $S_{\delta} \subset B^{\prime}$ from Theorem 7 and $\rho\left(S_{\delta}\right) \subset S_{\delta}$. Therefore the image $\rho^{p}\left(S_{\delta}\right)$ is disjoint with $\partial \mathcal{P}$ for all $p \geq 0$. So it is partitioned in $n$ disjoint pieces (may be some of them are empty) when intersecting $S_{\delta}$ with $\left\{B_{\{i\}}\right\}_{i \in I}$. In each of this pieces $\rho$ is continuous, and $\rho\left(S_{\delta}\right)=\bigcup_{i \in I} \rho\left(S_{\delta}\right) \cap B_{\{i\}}$.

Definition 10 Atoms of generation p. Given $i_{1} \in I$ we call atom of generation 1 to the following set:

$$
A_{i_{1}}=\rho\left(S_{\delta} \cap B_{\left\{i_{1}\right\}}\right)
$$

There are at least one and at most $n=\# I$ non empty atoms of generation 1 . Besides

$$
\rho\left(S_{\delta}\right)=\bigcup_{i_{1} \in I} A_{i_{1}} .
$$

Given $\left(i_{1}, i_{2}\right) \in I \times I$ we call atom of generation 2 to the following set: $A_{i_{1}, i_{2}}=\rho\left(A_{i_{1}} \cap\right.$ $\left.B_{\left\{i_{2}\right\}}\right)$.

There are at least one and at most $n^{2}=(\# I)^{2}$ non empty atoms of generation 2. Besides $\rho^{2}\left(S_{\delta}\right)=\bigcup_{\left(i_{1}, i_{2}\right) \in I^{2}} A_{i_{1}, i_{2}}$.

By induction, if defined the atoms $\left\{A_{i_{1}, i_{2}, \ldots, i_{p}}\right\}_{\left(i_{1}, \ldots, i_{p}\right) \in I^{p}}$

of generation $p$, we define the atoms of generation $p+1$ :

$$
\begin{aligned}
A_{i_{1}, i_{2}, \ldots, i_{p}, i_{p+1}} & =\rho\left(A_{i_{1}, i_{2}, \ldots, i_{p}} \cap B_{\left\{i_{p+1}\right\}}\right) \\
\text { Then: } \rho^{p}\left(S_{\delta}\right) & =\bigcup_{\left(i_{1}, i_{2}, \ldots, i_{p}\right) \in I^{p}} A_{i_{1}, i_{2}, \ldots, i_{p}} .
\end{aligned}
$$

There are at least one and at most $n^{p}=(\# I)^{p}$ non empty atoms of generation $p$. Fix $p \geq 1$. Denote $\mathcal{A}_{p}$ to the finite collection of atoms of generation $p: \mathcal{A}_{p}=\left\{A_{i_{1}, i_{2}, \ldots, i_{p}}\right\}_{\left(i_{1}, \ldots, i_{p}\right) \in I^{p}}$.

Remark: It is easy to check from the Definition above that for all $p \geq 1$, if $A \in \mathcal{A}_{p+1}$ then there exists $A^{\prime} \in \mathcal{A}_{p}$ such that $A \subset A^{\prime}$. Precisely $A=A_{i_{1}, i_{2} \ldots, i_{p+1}} \subset A_{i_{2}, \ldots, i_{p+1}}=A^{\prime}$

We define the diameter $d_{p}$ of the atomization of generation $p: d_{p}=\max _{A \in \mathcal{A}_{p}} \operatorname{diam}(A)$ where $\operatorname{diam}(A)=\sup _{V, W}\|V-W\|$ denotes the diameter of the atom $A$ and it is 0 if $A=\emptyset$. We observe that $d_{p}$ is not the diameter of the union of the atoms, even if they intersect.

Lemma 11 Let $0<\lambda<1$ the uniform contraction rate of Theorem 4 . There exists a constant $K$ such that for any $p \geq 1$ the diameter $d_{p}$ of the atomization of generation $p$ verifies:

$$
0 \leq d_{p} \leq K \lambda^{p-1}, \quad \lim _{p \rightarrow+\infty} d_{p}=0 .
$$


Proof: Take $K=\operatorname{diam} B$. Let us prove the thesis by induction on $p \geq 1$.

An atom $A \in \mathcal{A}_{1}$ of generation 1 is the image by $\rho$ of a set in $B$. Then $\operatorname{diam}(A) \leq K$. Suppose that all the atoms of generation $p$ have diameter smaller than $K \lambda^{p-1}$. Let us prove the thesis for $p+1$. An atom $A \in \mathcal{A}_{p}, p \geq 1$ is the image by $\rho$ of some set in $B$, so $A \subset \rho(B)$. Take an atom $A^{\prime} \in \mathcal{A}_{p+1}$. By definition: $A^{\prime}=\rho\left(A \cap B_{\{i\}}\right)$ for some $A \in \mathcal{A}_{p}$ and some $i \in I$.

As $A \cap B_{\{i\}} \subset \rho(B) \cap B_{\{i\}}$ due to Theorem $4 \rho$ is contractive there:

$$
\begin{gathered}
\operatorname{diam}\left(A^{\prime}\right)=\sup _{V^{1}, V^{2} \in A^{\prime}}\left\|V^{1}-V^{2}\right\|= \\
=\sup _{U^{1}, U^{2} \in A \cap B_{\{i\}}}\left\|\rho\left(U^{1}\right)-\rho\left(U^{2}\right)\right\| \leq \\
\leq \sup _{U^{1}, U^{2} \in A \cap B_{\{i\}}} \lambda\left\|U^{1}-U^{2}\right\|= \\
=\lambda \operatorname{diam}\left(A \cap B_{\{i\}}\right) \leq \lambda \operatorname{diam}(A) \leq \\
\leq K \lambda\left(\lambda^{p-1}\right)=K \lambda^{p} .
\end{gathered}
$$

Recall the distance $\operatorname{dist}(V, A)$ of a point $V \in B$ to a non empty set $A \subset B$ : $\operatorname{dist}(V, A)=$ $\sup _{W \in A}\|V-W\|$. Denote $\bar{A}$ and $\partial A$ to the closure and frontier respectively, of the set $A$. A basic classic result from Topology asserts that, in any compact connected metric space, the following properties hold, if $V \notin A$ : $\operatorname{dist}(V, A)=\operatorname{dist}(V, \bar{A})=\operatorname{dist}(V, \partial A)=$ $\max _{W \in \partial A}\|V-W\|=\left\|V-U_{0}\right\|$ for some $U_{0} \in \partial A$.

Lemma 12 If $V \in S_{\delta}$ then $\operatorname{dist}(V, \overline{\partial \mathcal{P}}) \geq \delta / 2$.

Proof: Due to the metric properties in compact connected spaces recalled above, it is enough to show that $\operatorname{dist}(V, \partial \mathcal{P}) \geq \delta / 2$. By contradiction suppose that $\inf _{U \in \partial \mathcal{P}}\|V-U\|<$ $\delta / 2$. Then there exists $U \in \partial \mathcal{P}$ such that: $\quad\|V-U\|<\delta / 2$.

$V \in B_{\delta}$ and by definition of uniform stability, if $\|V-W\|<\delta$ then:

$$
\left\|\rho^{k}(V)-\rho^{k}(W)\right\| \leq \alpha \quad \forall k \geq 1
$$

In particular $\|\rho(V)-\rho(U)\| \leq \alpha$.

On the other hand $U \in \partial \mathcal{P}$. Applying Theorem 7 there exists $W \in B$ such that

$$
\|U-W\|<\delta / 2, \quad\|\rho(U)-\rho(W)\|>3 \alpha .
$$

Joining the inequalities above with the triangular property: $\|V-W\| \leq\|V-U\|+\| U-$ $W \|<\delta$,

$$
\begin{aligned}
& 3 \alpha<\|\rho(U)-\rho(W)\| \leq \\
& \leq\|\rho(U)-\rho(V)\|+\|\rho(V)-\rho(W)\| \leq \\
& \leq \alpha+\|\rho(V)-\rho(W)\|, \\
& \|V-W\|<\delta, \quad 2 \alpha \leq\|\rho(V)-\rho(W)\| \text {. These last two inequalities contradict (16). }
\end{aligned}
$$

Lemma 13 If $V \in S_{\delta^{\prime}} \cap B_{\{i\}}$, then for all $W \in B$ such that $\|W-V\|<\delta / 2$ it is verified $W \in B_{\{i\}}$.

Proof: Consider the compact set $K=B \backslash B_{\{i\}}$. Then:

$U \in K \Leftrightarrow U \notin B_{\{i\}}$.

We have $V \notin K$. Call $d=\operatorname{dist}(V, K)=\inf _{U \notin B_{\{i\}}}\|V-U\|$. As recalled when defining the distance of a point to a set, there exists $U_{0} \in \partial K=\partial U_{\{i\}} \subset \overline{\partial \mathcal{P}}$ such that 


$$
d=\left\|V-U_{0}\right\|=\operatorname{dist}(V, \partial K) \geq \operatorname{dist}(V, \overline{\partial \mathcal{P}}) .
$$

Due to Lemma $12 \operatorname{dist}(V, \overline{\partial \mathcal{P}}) \geq \delta / 2$. In resume we have proved that: $d \geq \delta / 2, \quad \inf _{U \notin B_{\{i\}}} \| V-$ $U \| \geq \delta / 2$ Therefore, if $\|V-W\|<\delta / 2$ then $W \in B_{\{i\}}$.

Definition. Indivisibility of the atoms. An atom $A \in \mathcal{A}_{p}$ is indivisible if its closure $\bar{A} \subset B_{\{i\}}$ for some $i \in I$. From the definition of atom, $\rho(A)$ is a unique atom of generation $p+1$, i.e. $A$ does not divide when applying $\rho$. Besides $\rho$ is continuous in $B_{\{i\}}$ so, it is continuous in $\bar{A}$. Besides, $A \in \rho(B)$, so applying Theorem 4 ;

If an atom $A$ is indivisible then $\rho$ is continuous and uniformly contractive in $\bar{A}$.

\section{Proof of Theorem 9;}

By Lemma 11 there exists a generation $p \geq 1$ of atoms such that the diameter $d_{p}<\delta / 2$. Applying Lemma 13 and recalling that the diameter of a set is the same diameter of its closure, each atom $A \in \mathcal{A}_{p}$ is indivisible. From the remark at the end of Definition 10 the atoms of generation $p+1$ and later are contained in the atoms of generation $p$. Then all of them are also indivisible.

Fix some non empty atom $A=A^{1} \in \mathcal{A}_{p}$. As it is indivisible: $\emptyset \neq \rho\left(A^{1}\right) \in \mathcal{A}_{p+1}$. But any atom of generation $p+1$ is contained in an atom of generation $p$, so there exists $A^{2} \in \mathcal{A}_{p}$ such that $\rho\left(A^{1}\right) \subset A^{2}$. From the indivisibility, $\rho$ is continuous in $A^{1}$, thus: $\rho\left(\bar{A}^{1}\right) \subset \bar{A}^{2}$. Applying the same argument to $A_{2}$ instead of $A_{1}$ there exists $A^{3} \in \mathcal{A}_{p}$ such that $\rho\left(\bar{A}^{2}\right) \subset \bar{A}^{3}$. We deduce $\rho^{2}\left(\bar{A}^{1}\right) \subset \rho\left(\bar{A}^{2}\right) \subset \bar{A}^{3}$ where $A^{1}, A^{2}, A^{3} \in \mathcal{A}_{p}$. The family $\mathcal{A}_{p}$ is finite, so there is some first pair of integer numbers $k_{0} \geq 1, r_{0} \geq 1$ such that $A^{k_{0}}=A^{r_{0}+k_{0}} \in \mathcal{A}_{p}$. We conclude that some subfamily of $\mathcal{A}_{p}$ is related in a finite chain:

$$
\begin{array}{r}
\rho\left(\bar{A}^{1}\right) \subset \bar{A}^{2}, \ldots, \rho\left(\bar{A}^{j}\right) \subset \bar{A}^{j+1}, \ldots, \\
\ldots \rho\left(\bar{A}^{k_{0}}\right) \subset \bar{A}^{k_{0}+1}, \ldots, \\
\ldots \rho\left(\bar{A}^{k_{0}+r_{0}-1}\right) \subset \bar{A}^{k_{0}+r_{0}}=\bar{A}^{k_{0}} \\
\rho^{r_{0}}\left(\bar{A}^{k_{0}}\right) \subset \bar{A}^{k_{0}}
\end{array}
$$

All these atoms are indivisible by construction, so for each $\bar{A}^{j}, \mathrm{~J}=1,2 \ldots, \leq r_{0}-1$ in the finite chain (17), the Poincaré map $\rho: \overline{A^{j}} \mapsto \overline{A^{j+1}}$ is continuous and contractive, with a uniform contraction rate $0<\lambda<1$. In resume we have $\rho^{r_{0}}: \bar{A}^{k_{0}} \mapsto \bar{A}^{k_{0}}$ and $\left\|\rho^{r_{0}}(V)-\rho^{r_{0}}(W)\right\| \leq \lambda^{r_{0}}\|V-W\|$ for all $V, W \in \bar{A}^{k_{0}}$.

The Banach Fixed Point Theorem (Lages Lima [1970]), states that in any compact metric space $M$, given a uniformly contractive map $f$ such that $f(M) \subset M$, there exists and is unique a point $x_{1} \in M$ fixed by $f: f\left(x_{1}\right)=x_{1}$. Besides all orbits by future iterates of $f$ have limit $\left\{x_{1}\right\}$, i.e. $\lim _{k \rightarrow+\infty} f^{k}(x)=x_{1}, \forall x \in M$. We conclude that $\rho^{r_{0}}$ in the compact metric space $\overline{A^{k_{0}}}$ has a fixed point $V^{1}$ such that:

$$
\begin{aligned}
& V^{1} \in \overline{A^{k_{0}}}, \quad \rho^{r_{0}}\left(V^{1}\right)=V^{1}, \\
& \lim _{k \rightarrow+\infty} \rho^{k r_{0}}(V)=V_{1} \quad \forall V \in \overline{A^{k_{0}}}
\end{aligned}
$$

Therefore $V^{1}$ is periodic by $\rho$ of period $r_{0}$, and its orbit $L_{1}=\left\{V^{1}, \rho\left(V^{1}\right), \ldots, \rho^{r_{0}-1} V^{1}\right\}$ is the omega limit of all the points in $\bigcup_{j=1}^{j=k_{0}+r_{0}} \bar{A}^{j}$, in particular of those in $\bar{A}^{1}$.

From the definition of atom and from $\rho\left(S_{\delta}\right) \subset S_{\delta}$ each atom is contained in $S_{\delta}$. By Lemma 13 and using that $\rho$ is contractive in each of its continuity pieces intersected with $\rho(B)$, we deduce that all the points $W \operatorname{such}$ that $\operatorname{dist}\left(\rho(W), \bigcup_{j=k_{0}+1}^{k_{0}+r_{0}} \bar{A}^{j}\right)<\delta / 2$ have $\omega(W)=L_{1}$. 
Such points $W$ form an open set $\mathcal{N} \supset \bigcup_{j=k_{0}}^{k_{0}+r_{0}-1} \bar{A}^{j}$. Besides $L_{1} \subset \bigcup_{j=k_{0}}^{k_{0}+r_{0}-1} \bar{A}^{j}$. Then $\mathcal{N}$ is a neighborhood of $L_{1}$. Therefore the basin of attraction $B\left(L_{1}\right)$ of the periodic orbit $L_{1}$ contains a neighborhood of itself, verifying the definition of limit cycle.

In particular, $B\left(L_{1}\right) \supset \bar{A}^{1} \in \mathcal{A}_{p}$. The construction above can be done choosing any first atom $A_{1} \in \mathcal{A}_{p}$. We deduce that for each $A \in \mathcal{A}_{p}$ there exists a limit cycle $L \subset B^{\prime}$ such that $\bar{A} \subset B(L)$.

Define $\mathcal{L}=\left\{L \subset B: L\right.$ is a limit cycle, $A \subset B(L)$ for some $\left.A \in \mathcal{A}_{p}\right\}$. It is a finite and not empty collection because the family $\mathcal{A}_{p}$ of the atoms of generation $p$ is finite and not empty. By construction the union of the basin of attractions of all the limit cycles in $\mathcal{L}$ contains all the atoms of generation $p$. Therefore:

$\rho^{p}\left(B_{\delta}\right)=\bigcup_{A \in \mathcal{A}_{p}} A \subset \bigcup_{L \in \mathcal{L}} B(L)$. For all $U$ the limit set $\omega(U)=\omega\left(\rho^{p}(U)\right)$. Then $B_{\delta} \subset$ $\bigcup_{L \in \mathcal{L}} B(L)$.

\section{CONCLUSIONS}

We described and analyzed a general mathematical model of a network of $n$ inhibitory pacemaker neurons interacting by synapsis without delay. The size of the network must be finite but as large as wanted. We found discontinuities in the dynamical system due to the synaptic coupling and proved that they imply the existence of chaotic orbits. The Poincaré return map $\rho$ to the $(n-1)$-dimensional section $B$ in the phase space, results from considering the state of the system immediately after each spike. We proved topological and measure properties of $\rho$ as mathematical tools to obtain the dynamical results. We classified the systems according to the measure of the chaotic set and proved that, even being this set never empty, if it has not full measure there exist stable points and thus, limit cycles. It is unknown if there exist $\mathcal{C}^{1}$ systems of this model exhibiting a set of chaotic orbits with positive measure, although under some other additional hypothesis, there are known results about the genericity of the systems exhibiting only limit cycles.

\section{Acknowledgments}

We thank the project PDT 54/001 of Clemente Estable found (Uruguay), the University of Valparaíso (Chile) and the University of Marburg (Germany) for partial support, Profs. Pierre Guiraud, Hans Braun and Ruben Budelli for their suggestions, and MEDYFINOL organizing \& scientific comitee for its invitation.

\section{REFERENCES}

Abbott, L.F. \& Vreeswijk. C [1993] "Assynchronous states in neural networks of pulsecoupled oscillators." Phys. Rev. E 48 1483-1490

Bessloff, P. \& Coombes, S. [2000] "Dynamics of Strongly Coupled Spiking Neurons." Neural Computation 12 91-129.

Budelli, R. , Catsigeras, E. , Rovella, A. \& Gómez, L. [1996] "Dynamical behavior of pacemaker neurons networks." Proc. of the Second Congress of Nonlinear Analysts, Elsevier Science.

Budelli, R., Torres, J., Catsigeras, E. , \& Enrich, H. [1991] " Two neurons networks I: Integrate and fire pacemakers models." Biol. Cybern.66, 95-110

Catsigeras, E. \& Budelli, R. [1992] "Limit cycles of a bineuronal network model. " Physica D.56, 235-252 
Catsigeras, E., Rovella, A. \& Budelli, R. [2008] " Contractive piecewise continuous maps modeling networks of inhibitory neurons." ArXiv [q-bio $N C] \mathbf{0 8 0 5 . 2 6 9 5 v 1}$

Cessac, B. [2008] "A discrete time neural network model with spiking." J. Math. Biol. 54, 311-345

Cessac, B. \& Viéville, T. [2008] "On Dynamics of integrate and fire neural networks with conductance based synapses." ArXiv [phys-bio-ph] 0709.4370v3

Lages Lima, E. [1970] "Elementos de Topologia Geral." Projeto Euclides, I.M.P.A. , Rio de Janeiro.

Mirollo, R.E. \& Strogatz, S.H. [1990] "Synchronisation of pulse coupled biological oscillators" SIAM, J. Appl. Math. 50 1645-1662

Postnova, S., Wollweber, B., Voigt, K., Braun, H. A. [2007] "Neural Impulse Pattern in Bidirectionally Coupled Model of Neurons of Different Dynamics." Biosystems 89 135-142

Rey Pastor, J., Pi Calleja P., Trejo C. [1968] "Análisis Matemático. Vol. II. Cálculo infinitesimal de varias variables." Ed. Kapelusz. Buenos Aires.

Sotomayor, J. [1979] "Liçòes de equaçoes diferenciais ordinárias." Projeto Euclides, I.M.P.A., Rio de Janeiro. 